\title{
The reading strategies of a map-based dashboard - acquisition and assessment
}

\author{
Chenyu Zuo a, Linfang Ding ${ }^{\mathrm{b},}$, , Liqiu Meng ${ }^{\mathrm{a}}$ \\ ${ }^{a}$ Chair of Cartography, Technical University of Munich, 80333 Munich, Germany, chenyu.zuo@tum.de; Liqiu Meng, \\ liqiu.meng@tum.de \\ ${ }^{b}$ KRDB Research Centre, Faculty of Computer Science, Free University of Bozen-Bolzano, 39100 Bozen-Bolzano, Italy, \\ linfang.ding@unibz.it \\ * Corresponding author
}

Keywords: user study, map-based dashboard, interview, eye-tracking

\begin{abstract}
:
Map-based dashboard represents an intuitive tool that can enable novices for various purposes, such as urban environment learning, pandemic monitoring, and education (Häägg et al. 2020). Maps are embedded to highlight the spatial information in combination with multiple charts and texts to give users an overview of the shown topics. However, little is known about how users read map-based dashboard to gain various insights. This study aims to assess users' reading strategies of map-based dashboards. We combine the methods of interview with eye-tracking to externalize reading strategies for map-based dashboards designed for a specific purpose (Holmqvist et al. 2011; Beitlova et al. 2020). The results can be used to guide the layout and interface design of future map-based dashboards.
\end{abstract}

Participants. 40 gender-balanced participants with diverse background will be recruited in this study. All the participants should be above 18 years old. In addition, we will try to recruit an equal number of map-reading experts and novices. Another grouping criteria will be their knowledge level about the mapped area between no (never heard of it), some (heard of it), much (visited it) and on site (lives there).

Material. We will use the map-based dashboard designed for learning and analyzing an industrial innovation environment in the area of Jiangsu province, China. The dashboard contains seven panels as shown in Figure 1. Users may explore the performances of different cities based on the statistics and spatial distribution, influence factors and their correlations.

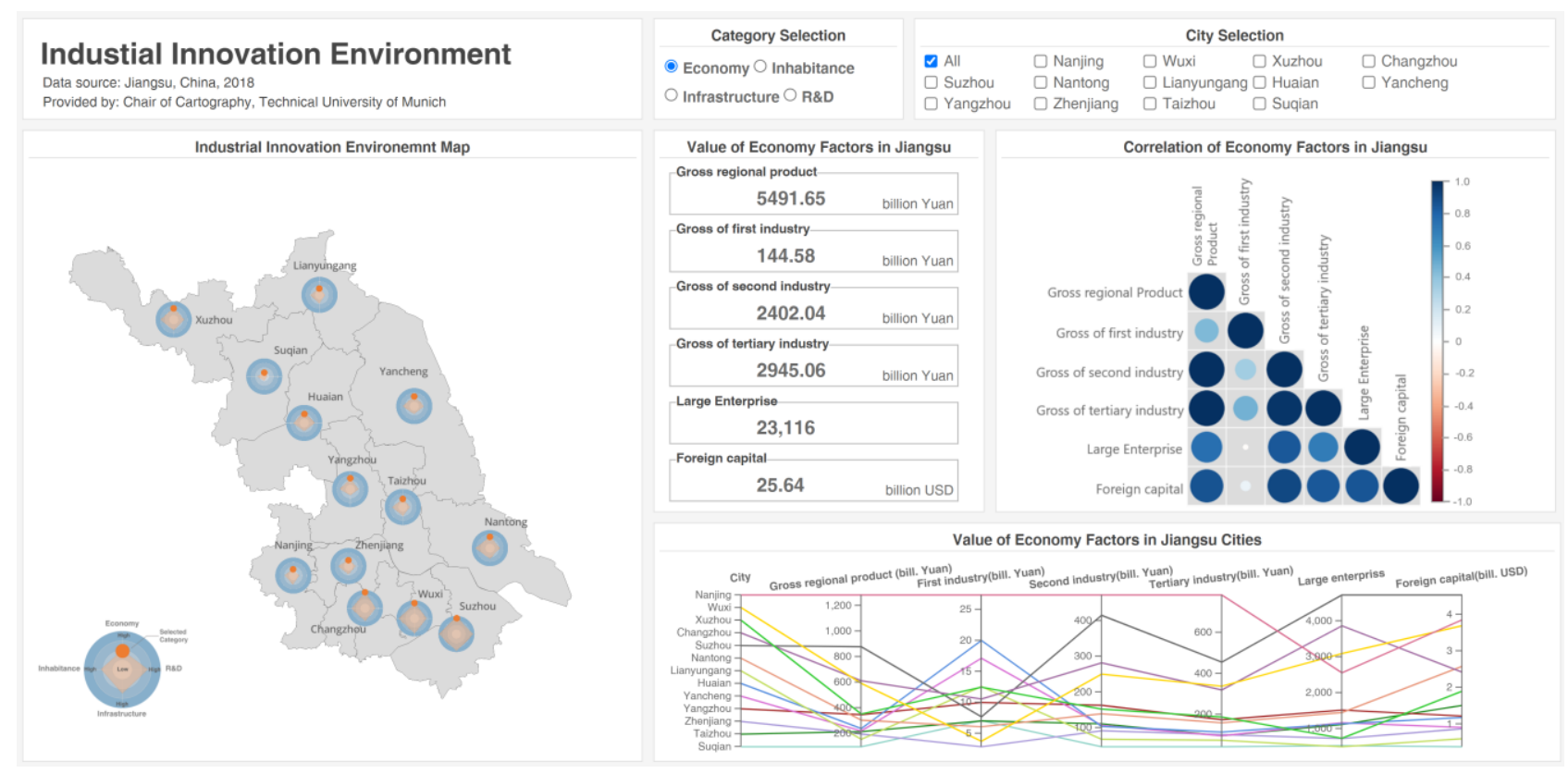

Figure 1. The layout of an interactive map-based dashboard used in our experiment.

Tasks. As shown in the following table, six tasks are designed to represent the insights at different difficulty levels including fact finding at different aggregation levels, comparison of factors and cities, identify correlations, and summarize the industrial innovation environment in different areas. 


\begin{tabular}{ll}
\hline Tasks & Description \\
\hline Task01 & Find the population of the Jiangsu Province. \\
Task02 & Find the GDP of Wuxi. \\
Task03 & Find the correlation between the factor number of students and experts. \\
Task04 & Find the city with the highest value of technology index. \\
Task05 & Describe the social factors in Xuzhou. \\
Task06 & Describe the infrastructure in Jiangsu province. \\
\hline
\end{tabular}

Procedure. The experiment will be conducted in the eye-tracking lab at the Technical University of Munich. The participants will be individually guided throughout the experiment. First, they are introduced to map-based dashboard as a cartographic tool and its design purposes. Upon consent, their demographic information about gender, age, expertise of maps, and knowledge about the mapped area is collected, and the eye-tracker is calibrated with the participants. Once prepared, the participants perform the six tasks with their eye movements tracked. The screen with the eye movement paths is recorded as videos. Finally, we interview the participants about their reading strategy when they were performing the tasks. The recorded video will be provided to the participants to help them recall their reading strategies.

The reading strategies of map-based dashboard can be derived from the interview protocols. In particular, the function of maps, charts, titles, and interactions will be determined. The satisfaction with the arrangement of panels can also be inferred from the interviews. We expect that the results will help future designers of map-based dashboards to improve the layout design, which can better guide users to gain important insights.

\section{References}

Fredriksson Häägg, A., Weil, C., \& Rönnberg, N. (2020). On the usefulness of map-based dashboards for decision making.

Beitlova M, Popelka S, Vozenilek V. Differences in Thematic Map Reading by Students and Their Geography Teacher. ISPRS International Journal of Geo-Information. 2020; 9(9):492. https://doi.org/10.3390/ijgi9090492

Holmqvist, K., Nyström, M., Andersson, R., Dewhurst, R., Jarodzka, H., \& Van de Weijer, J. (2011). Eye tracking: A comprehensive guide to methods and measures. OUP Oxford. 